\title{
Cytogenetic biological dosimetry assays: recent developments and updates
}

\author{
Tamizh Selvan Gnanasekaran \\ Central Research Lab, K. S. Hegde Medical Academy, Nitte (Deemed to be University), Mangalore, India
}

Received: March 11, 2021

Revised: April 16, 2021

Accepted: April 21, 2021

Correspondence:

Tamizh Selvan Gnanasekaran

Central Research Lab., K. S. Hegde

Medical Academy, Nitte (Deemed to

be University), Nityanandanagar,

Deralakatte, Mangalore 575018, India

Tel: +96-29876235

E-mail:drtamizhselvang@nitte.edu.in ORCID:

https://orcid.org/0000-0001-8774-2442
Biological dosimetry is the measurement of radiation-induced changes in the human to measure short and long-term health risks. Biodosimetry offers an independent means of obtaining dose information and also provides diagnostic information on the potential for "partial-body" exposure information using biological indicators and otherwise based on computer modeling, dose reconstruction, and physical dosimetry. A variety of biodosimetry tools are available and some features make some more valuable than others. Among the available biodosimetry tool, cytogenetic biodosimetry methods occupy an exclusive and advantageous position. The cytogenetic analysis can complement physical dosimetry by confirming or ruling out an accidental radiological exposure or overexposures. We are discussing the recent developments and adaptability of currently available cytogenetic biological dosimetry assays.

Keywords: Biological dosimetry, Cytogenetics, Radiation

\section{Introduction}

In each phase, a human being's lifespan is considered to be exposed to ionizing radiation (IR) because of its various applications. They are characterized as ionizing based on the distinct photon's energy; those are $X$-rays, $\gamma$-rays, $\alpha$-particles, $\beta$-particles, and neutron. IR has numerous prospective applications such as medical diagnosis, cancer treatment, power generation, etc. It is inevitable that a definite amount of radiation exposure to humans while implementing IR for those beneficial applications. Nevertheless, because of the support of IR and reported side effects, the use of radiation application is decisively controlled, measured and monitored by the regulatory bodies. Those are the International Atomic Energy Agency (IAEA), the International Commission on Radiological Protection (ICRP), the World Health Organization (WHO) and the United Nations Scientific Committee on the Effects of Atomic Radiation (UNSCEAR) for assessing radiation risks, provide recom- mendations, and promote the safe use of radiation technologies, which is developing rapidly and gaining complexity. In addition to occupational exposure, accidental, radiological or nuclear exposure can cause serious health effects. More than that, radiological or nuclear terrorism constitutes a potential threat to several nations, where several hundred and thousands of people may potentially get exposed to radiation. Many atomic disasters since Chernobyl [1] to Fukushima Daiichi [2] clearly illustrate a critical need for suitable biomarkers for personalized radiation dose assessment where physical dosimeters are unavailable, which can be useful for appropriate medical/clinical management.

The estimation of the dose received by an individual during accidental exposure, suspected overexposures, and the fraction of body exposed and dose to the irradiated fraction using a biological indicator is termed biological dosimetry. It is observed that recording various clinical signs such as cell counts in blood, functions of the central nervous system, vomiting, diarrhea, and alopecia as acute

Copyright (C) 2021 The Korean Society for Radiation Oncology

This is an Open Access article distributed under the terms of the Creative Commons Attribution Non-Commercial License (http://creativecommons.org/licenses/by-nc/4.0/) which permits unrestricted non-commercial use, distribution, and reproduction in any medium, provided the original work is properly cited. 
radiation syndrome using biological dosimetry [3]. These preliminary assessment results would enlighten the exposure dose range and therapeutic management of that incident. Moreover, biological dosimetry directs and offers assessment provision for screening, therapeutic management, and long-term risk assessment. The radiation-induced changes in biomolecules can be measured using biological indicators. There are many biological indicators of radiation exposure, such as cytogenetics [4], mutations [5], gene expression [6], protein modifications, such as $\gamma-\mathrm{H} 2 \mathrm{AX}$ [7], metabolic intermediates [8], inflammatory signaling [9], genomics [10], transcriptomics [11], and proteomics [12]. Out of these mentioned biological indicators, cytogenetic biomarkers (Table 1) are considered the most sensitive and reliable among the biological indicators used to quantify an absorbed radiation dose during radiation emergencies. After cells getting exposed to radiation, DNA strand breaks take place and by the cellular DNA repair systems, which are then repaired. Unrepaired and poorly repaired breaks can lead to abnormal changes in chromosome structures and form dicentric chromosomes (DC), containing two centromeres after fusion of two damaged chromosomes and broken chromosome rearrangements result in translocation. Counting these various abnormal chromosomes is dose-related, providing a strong dose-response relationship [13]. The strategy for growing the throughput of biological dosimetry is developing networks among biodosimetry laboratory available globally. Several networks have been recognized to improve dose estimation throughput, such as the Latin American Biological Dosimetry Network [14], the National Biological Dosimetry Response Plan (NBDRP) in Canada [15], the Chromosome Network in Japan [16], BioDoseNet [17], the European Network, Realizing the European Network of Biodosimetry (RENEB) [18], NATO biodosimetry group [19], EURADOS (European Radiation Dosimetry Group) [20], China [21] and in South Korea [22] for the cytogenetic assays.

\section{Cytogenetic Biodosimetry Assays}

\section{DC assay}

It is essential to quantify aberrations in first-division cycle metaphase spreads for cytogenetic biological dosimetry using human peripheral blood lymphocytes. Structural changes are observed in their entirety without the confounding effects of elimination and dilution of aberrations associated with cell division. The most commonly occurring radiation-induced abnormality is DC. The DC is an aberrant chromosome with two centromeres formed by the fusion of two different chromosome segments, each with a centromere, which accompanies an acentric fragment. The DC assay, which has to be performed after 2 days of whole blood culture with phytohemagglutinin, followed by a 24-hour incubation with colchicine,

Table 1. Comparison of cytogenetic biodosimetry assays

\begin{tabular}{|c|c|c|c|c|}
\hline & Dicentric & CBMN & Translocations & PCC \\
\hline Detection limit (Gy) & 0.1 & 0.25 & 0.30 & 0.05 \\
\hline Culture time (hr) & 48 & 72 & 72 & 2 (fusion time) \\
\hline Inter-laboratory comparison & $\checkmark$ & $\checkmark$ & $\checkmark$ & $\checkmark$ \\
\hline Automation & $\checkmark$ & $\checkmark$ & $\checkmark$ & $\checkmark$ \\
\hline Advantages & $\begin{array}{l}\text { Gold standard } \\
\text { Specific to ionizing radiation } \\
\text { Low baseline frequency } \\
\text { Accepted world-wide }\end{array}$ & $\begin{array}{l}\text { Less time required } \\
\text { Non-technical person can be } \\
\text { scored }\end{array}$ & Retrospective analysis & $\begin{array}{l}\text { Does not require culture time } \\
\text { Can be detected }>5 \mathrm{~Gy}\end{array}$ \\
\hline Disadvantages & $\begin{array}{l}\text { Time consuming } \\
\text { Technical expertise required }\end{array}$ & $\begin{array}{l}\text { Non-specific to radiation } \\
\text { Baseline variation }\end{array}$ & $\begin{array}{l}\text { More expensive (mFISH/SKY) } \\
\text { Highly expertise dependent } \\
\text { Need quality fluorescent mi- } \\
\text { croscope }\end{array}$ & $\begin{array}{l}\text { Time consuming } \\
\text { Technical expertise required }\end{array}$ \\
\hline \multicolumn{5}{|l|}{ Developments } \\
\hline Networking & $\checkmark$ & $\checkmark$ & $\checkmark$ & $\checkmark$ \\
\hline Web based scoring & $\checkmark$ & $\checkmark$ & $\checkmark$ & $\checkmark$ \\
\hline Dose assessment for triage & $\checkmark$ & $\checkmark$ & $x$ & $\checkmark$ \\
\hline Triage scoring (50 cells) & $\checkmark$ & $x$ & $x$ & $\checkmark$ \\
\hline RABiT & $\checkmark$ & $\checkmark$ & $x$ & $x$ \\
\hline Partial-body exposures & $\checkmark$ & $x$ & $x$ & $\checkmark$ \\
\hline
\end{tabular}

CBMN, cytokinesis blocked micronucleus; PCC, premature chromosome condensation; RABiT, Rapid Automated Biodosimetry Tool; mFISH/SKY, multiple fluorescence in situ hybridization/spectral karyotyping. 
a mitosis-blocking agent. After fixation, metaphase chromosomes are generally stained with Giemsa, representing the conventional method and centromeric staining allows better detection of chromosomal aberrations [23]. DCs are sensitive for agents that induce double-stranded DNA breaks, such as IR. They can be used to estimate the unknown absorbed dose during a radiological emergency by counting their frequency. Furthermore, DC's are unstable aberrations, and during mitotic division, it can induce cell death, limiting the use of this biomarker to a post-exposure after few months. The background frequency is 1 in 1,000 cells irrespective of ages, and the sensitivity of the DC test is $0.1 \mathrm{~Gy}$, making it the gold standard for biodosimetry applications [24]. The DC assay has been widely used in numerous accidental events, such as Chernobyl [25], Istanbul [26], Goiania [27], Bangkok [28], Marshall Islands [29], Taiwan [30], and Xinzhou, China [31]. However, the analysis is long and laborious. It requires qualified personnel to identify chromosomal aberrations in the course of large scale radiological incidents, where rapid segregation is vital for triage and needs to be segregated by no exposure, moderately or severely exposed humans to obtain suitable therapeutic countermeasures. In this view, to speed up the scoring and confirm clinical triaging can typically be achieved by scoring 20 metaphases per subject. If there is disagreement, scoring should be increased to 50 cells [32]. The evidence from published data [33] shows that the automated DC scoring positively improves the rapidity of radiation absorbed dose assessment. And also, a single lab cannot handle the enormous number of samples and it is essential to join hands with other labs in and around their region. For this, an exercise was initiated, such as inter-lab comparison, which gives knowledge on harmonization in the protocol, sharing the workload, scoring criteria. The investigation on chromosome preparations or analysis by microscopic analysis and/or image galleries made by other laboratories is vastly improved via inter-lab comparison exercises worldwide [14-16,23,34]. While some aspects of the assay have been successfully streamlined, the overall throughput remains limited by the labor-intensive identification of DCs in many cells. This affects the timely estimation of radiation exposure, especially for testing multiple affected individuals in a large accident or a mass casualty nuclear event [35]. Conventional DC assay remains the "gold standard" biodosimetric technique in estimating the radiation absorbed dose because of its specificity, sensitivity and low background frequency. In the case of mass casualty radiology or nuclear events, several thousands of people may get exposed. In such situations, the DC analysis for such a large population will take a considerable amount of time. Its long assay time (> 50 hours) makes it impractical for its application during mass casualty incidents. During triage, to reduce the scoring burden, to improve the time efficiency and without los- ing accuracy, a scoring technique called "DCA QuickScan" [36] has been introduced dose estimation.

\section{Cytokinesis blocked micronucleus assay}

Micronuclei (MN) are formed from lagging chromosome fragments or whole chromosomes at anaphase that are not included in the daughter cell's nuclei. Therefore, they are seen as distinctly separate small spherical objects with the same morphology and staining properties of nuclei within the daughter cell's cytoplasm [37]. MN reflects chromosomal damage and is a useful index for monitoring environmental effects on human cell's genetic material [38]. Due to the simplicity and rapidity of scoring, this assay has shown promising potential in triage medical management. However, due to the background frequency of spontaneous MN frequency (0.002 to $0.036 /$ cells), the sensitivity is $0.25 \mathrm{~Gy}$ [24]. The assay was adopted in Chernobyl [39] and Istanbul [40] radiation accident. MN reflects chromosomal damage and is a useful indicator for monitoring environmental effects [39]. Even though these MN are not radiation specific, an increase with dose has been observed and validated as a technique for estimating radiation exposure [41]. Cytokinesis blocked micronucleus (CBMN) assay's advantage over the DC assay is that the analysis manually is considerably faster. Only 200 binucleated cells are required to provide a sensitivity of $1 \mathrm{~Gy}$ [42], and the method requires less technical expertise. In the case of large-scale accidents involving thousands of victims, a single biological dosimetry laboratory's capacity would be insufficient. A biodosimetry laboratory network's availability would be an essential element to ensure adequate emergency response strategies [17]. Thus, an effort has been made to establish a network that can provide a harmonized performance of biological dosimetry assays [43]. However, this approach involves sample shipment and tracking, which is often problematic. It is necessary to setting-up for high throughput to handle multiple samples in a large-scale situation. A biodosimetry laboratory engaging operational automation could take countless samples [44]. At present, several studies have confirmed the reliability of the automated CBMN assay for high throughput population triage [45-47].

The absence of cellular visualization using conventional flow cytometry makes it impossible to quantify mono-, bi-, and poly-nucleated cells with and without MN. To overcome this, imaging flow cytometry has been proposed, which combines high-resolution microscopy with flow cytometry. Cells in suspension can be captured, removing the need for microscope slides and allowing visualization of intact cytoplasmic membranes and DNA content. Thus, mono-, bi-, and poly-nucleated cells with and without MN can be rapidly and automatically identified and quantified [46]. Rapid Automated Biodosimetry Tool II (RABiT-II), a fully automated with a signifi- 
cantly reduced time to obtain the result for a fast high-throughput biodosimetric estimation, has been proposed. By using this approach, the radiation doses for irradiated samples from two donors were estimated within $20 \%$ of the correct dose $( \pm 0.5$ Gy below 2 Gy) in $97 \%$ of the samples, with the doses in some 5 Gy irradiated samples being underestimated by up to 25\% [47]. Recently, a quick CBMN harvest protocol has been proposed for isolated peripheral blood mononuclear cells and it is a viable alternative to cytocentrifugation, as many scorable binucleated cells were obtained with routine biodosimetry [48].

\section{Translocation Assay}

IR induced chromosomal exchanges like DC and MN are eliminated from the circulating blood within 1-3 years depending on the exposure. As a result, there is considerable uncertainty in dosimetry for past exposures [49]. Scoring stable chromosomal exchanges such as translocations is an alternative approach to overcome this problem. Translocations result from a minimum of two DNA DSBs and a union of at least two altered chromosomes. The method used for stable aberrations is currently the analysis of chromosomal translocations. Many laboratories initially explored G-banding's use to identify translocations for biodosimetry purposes [50]. The bands are formed on chromosomes when metaphase chromosomes are treated with a proteolytic enzyme, the trypsin. Banding allows identifying individual chromosomes and chromosomal regions within it and specific chromosome alterations like translocations, inversion, etc. This technique was employed to estimate the dose received by atomic bomb survivors [50] and long-term occupationally exposed radiation technologists [51]. It is possible to estimate the absorbed dose more than 20 years after exposure using in situ hybridization combined with various chromosome-staining strategies [52]. Fluorescence in situ Hybridization (FISH) allows detecting DNA sequences in chromosome preparations, including stable aberrations like translocations. A FISH assay-based translocation measurements are fast and straightforward compared to G-banding and superior to conventional DC analysis for retrospective biological dosimetry [24]. FISH's utility with whole-chromosome probes is a potential utility for translocation frequency analysis in assessing the level of acute radiation exposure independent of the time between analysis and exposure [53]. Guerrero-Carbajal et al. [54] reported the persistence of translocations through three cell cycles in irradiated cultures of lymphocytes. This assay has been used in follow-up study among Chernobyl accident victims from 1991 to 1994 [55]. The frequencies of translocations recorded in atomic bomb survivors and estimated doses were closer to the expected dose derived by calculation obtained with an in vitro dose-response curve [56]. FISH-based translocation analysis's main advantage is that the aberrations are dis- tinct and easy to discriminate between dicentrics and translocations. The yield of stable chromosomal aberrations was high, correlating with the severity of acute radiation sickness (ARS) in the blood cells of 17 radiation accident victims who had suffered ARS ranging from severity grades I to IV [57]. However, it is robust and seems to be less sensitive than the others, with a detection limit of approximately $0.30 \mathrm{~Gy}$, according to several studies [52]. The evaluation of this lack of sensitivity in low doses clarified that it is due to spontaneous translocations rate in individual variability, assessed in large-scale inter-laboratory exercises [58].

\section{Premature Chromosome Condensation Assay}

Usually, to study chromosomal aberrations, like DC assay in lymphocytes, it has to be stimulated to divide and then arrested at the metaphase stage. This procedure involves a turn-around time of $>50$ hours after blood withdrawal. In a radiation emergency, exposed individuals cannot wait until the results to start the medical treatment. Hence, the premature chromosome condensation (PCC) technique was first developed by Johnson and Rao [59]. The mitotic phase cells induce the condensation of chromatin filaments at interphase cells upon fusion with a fusogen. Conventionally, PCC is induced by fusing peripheral blood lymphocytes (PBL) with mitotic Chinese hamster ovary ( $\mathrm{CHO}$ ) cells using polyethylene glycol or Sendai virus [59]. In peripheral blood, most of the cells will be in the resting stage $(\mathrm{G} 0)$ during collection, so it is useful to estimate the dose using PCC. This technique helps to study the radiation-induced damages without stimulation of the cells. The aberrations can be studied within 2 hours; therefore, chances for the loss of information due to interphase cell death are reduced [60]. Another advantage of this assay is that it can be used at high dose (> $5 \mathrm{~Gy}$ ) estimation because conventional cytogenetic dosimetry using chromosomal aberrations becomes difficult due to mitotic delay and disappearance of lymphocytes from peripheral blood circulation [61]. It is proven that some accidents need to estimate exposures for high doses and whole or partial body exposures [62]. Recently, studies have shown that using this technique dose-effect curve can be generated for higher doses for gamma rays (25 Gy) and neutrons (10 Gy) [63-65]. In addition to using non-mitogen stimulated culture for the PCC assay, many researchers use mitogen-stimulated cultures (48 hours) for the PCC assay and selected G2PCC spreads to score structural chromosomal aberrations [66]. Many studies have investigated exposure biomarkers for high dose radiation. The dose-response of multiple biological endpoints, including G2/A-PCC (G2/M and M/A-PCC) index, PCC ring (PCC-R), a ratio of the longest/shortest length (L/L ratio), and length and width ratio of the longest chromosome (L/B ratio) has been reported [67]. However, skilled personnel must do the scoring as the 
metaphases contain chromosomes of both lymphocytes and mitotic cells. The minimum dose detection limit of using this technique is 0.05 Gy [68]. The PCC assay with PBL is recommended as a rapid biodosimetry method [69]. Biological dosimetry was performed on three seriously exposed victims of the Tokaimura criticality accident in Japan [70]. The use of fluorescent staining to detect centromeres and telomeres on PCC fragments has made it possible to count the anomalies typically identified (dicentrics, rings, acentric) [71]. Manual scoring following telomere and centromere staining revealed a significantly higher frequency of dicentrics (up to 30\%) compared to Giemsa staining due to improved detection of dicentrics with centromeres. This enhancement allowed improved software, TCScore, that detected 95\% of manually scored dicentrics compared to $50 \%$ for the best currently available software (DCScore) [71]. Blind dose assessments were executed for triage biodosimetry investigating G0-lymphocyte PCC for standardization, harmonization, and validating the PCC assay [72]. It is also possible for automation [73], in addition, and new approaches have been introduced [74]. The combination of automatic image acquisition and automated image analysis of PCC allows calculating the length ratio of the longest and the shortest chromosome pieces that can be used for dose estimation [66].

\section{Conclusion}

The point-of-care response plans need to be optimized for the medical management of mass casualty radiological events for triage management to integrate biodosimetry cytogenetic assays, validate these technologies through field testing and optimization of reach back diagnostic laboratory networks. However, DC assay remains the gold standard among all other cytogenetic assays employed for biological dosimetry. Whereas underestimation of dose would lead to analyzing unstable chromosome aberrations for retrospective dose estimation, stable aberrations like translocations would give accurate dose estimation of late exposure. Some other dose assessment modes using genomics, proteomics, and electron paramagnetic resonance (EPR) methods are in development. In future perspective, to avoid underestimating the dose due to variations in individual radiosensitivity, the best approach is probably to combine a cytogenetic test with physical dosimetry or even a targeted genomic approach, especially in the case of disease. Targeted genomics is booming, particularly in the field of cancer.

\section{Conflict of Interest}

No potential conflict of interest relevant to this article was reported.

https://doi.org/10.3857/roj.2021.00339

\section{References}

1. United Nations Scientific Committee on the Effects of Atomic Radiation. UNSCEAR 2008 Report: Sources and effects of ionizing radiation. Vienna, Austria: United Nations Scientific Committee on the Effects of Atomic Radiation; 2011.

2. World Health Organization. The Great East Japan Earthquake: a story of devastating natural disaster, a tale of human compassion. Manila, Philippines: World Health Organization; 2012.

3. Vorobiev Al. Acute radiation disease and biological dosimetry in 1993. Stem Cells 1997;15 Suppl 2:269-74.

4. Ramalho AT, Nascimento AC, Natarajan AT. Dose assessments by cytogenetic analysis in the Goiania (Brazil) radiation accident. Radiat Prot Dosim 1988;25:97-100.

5. Rigaud O, Laquerbe A, Moustacchi E. DNA sequence analysis of HPRT- mutants induced in human lymphoblastoid cells adapted to ionizing radiation. Radiat Res 1995;144:181-9.

6. Dressman HK, Muramoto GG, Chao NJ, et al. Gene expression signatures that predict radiation exposure in mice and humans. PLoS Med 2007;4:e106.

7. Moquet J, Barnard S, Rothkamm K. Gamma-H2AX biodosimetry for use in large scale radiation incidents: comparison of a rapid ' 96 well lyse/fix' protocol with a routine method. PeerJ $2014 ; 2:$ :282.

8. Pannkuk EL, Fornace AJ Jr, Laiakis EC. Metabolomic applications in radiation biodosimetry: exploring radiation effects through small molecules. Int J Radiat Biol 2017;93:1151-76.

9. Mukherjee S, Laiakis EC, Fornace AJ Jr, Amundson SA. Impact of inflammatory signaling on radiation biodosimetry: mouse model of inflammatory bowel disease. BMC Genomics 2019;20:329.

10. Robson T, West C. Radiation and the genome: from risks to opportunities for therapeutic exploitation. Br J Radiol 2010;83:6357.

11. Michna A, Schotz U, Selmansberger M, et al. Transcriptomic analyses of the radiation response in head and neck squamous cell carcinoma subclones with different radiation sensitivity: timecourse gene expression profiles and gene association networks. Radiat Oncol 2016;11:94.

12. Sharma M, Halligan BD, Wakim BT, Savin VJ, Cohen EP, Moulder JE. The urine proteome for radiation biodosimetry: effect of total body vs. local kidney irradiation. Health Phys 2010;98:186-95.

13. Herate C, Sabatier L. Retrospective biodosimetry techniques: focus on cytogenetics assays for individuals exposed to ionizing radiation. Mutat Res 2020;783:108287.

14. Garcia OF, Ramalho AT, Di Giorgio M, et al. Intercomparison in cytogenetic dosimetry among five laboratories from Latin America. Mutat Res 1995;327:33-9. 
15. Miller SM, Ferrarotto $C L$, Vlahovich $S$, Wilkins $R C$, Boreham DR, Dolling JA. Canadian Cytogenetic Emergency network (CEN) for biological dosimetry following radiological/nuclear accidents. Int J Radiat Biol 2007;83:471-7.

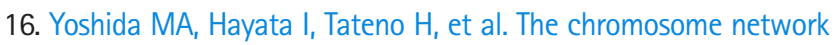
for biodosimetry in Japan. Radiat Meas 2007;42:1125-7.

17. Blakely WF, Carr Z, Chu MC, et al. WHO 1st consultation on the development of a global biodosimetry laboratories network for radiation emergencies (BioDoseNet). Radiat Res 2009;171:12739.

18. Kulka U, Ainsbury L, Atkinson $M$, et al. Realising the European Network of Biodosimetry (RENEB). Radiat Prot Dosimetry 2012;151:621-5.

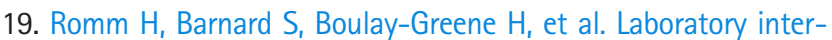
comparison of the cytokinesis-block micronucleus assay. Radiat Res 2013:180:120-8.

20. Ainsbury E, Badie C, Barnard S, et al. Integration of new biological and physical retrospective dosimetry methods into EU emergency response plans: joint RENEB and EURADOS inter-laboratory comparisons. Int J Radiat Biol 2017;93:99-109.

21. Pan Y, Ruan J, Gao G, Wu L, Piao C, Liu J. Laboratory intercomparison of cytogenetic dosimetry among 38 laboratories in China. Dose Response 2019;17:1559325819833473.

22. Lee $Y$, Jin $Y W$, Wilkins $R C$, Jang $S$. Validation of the dicentric chromosome assay for radiation biological dosimetry in South Korea. J Radiat Res 2019;60:555-63.

23. Bhavani M, Tamizh Selvan G, et al. Dicentric chromosome aberration analysis using giemsa and centromere specific fluorescence in-situ hybridization for biological dosimetry: an inter- and intra-laboratory comparison in Indian laboratories. Appl Radiat Isot 2014;92:85-90.

24. International Atomic Energy Agency. Cytogenetic dosimetry: applications in preparedness for and response to radiation emergencies. Vienna, Austria: International Atomic Energy Agency; 2011, p. 59-79.

25. European Commission; International Atomic Energy Agency; World Health Organization. One decade after Chernobyl: summing up the consequences of the accident. Vienna, Austria: International Atomic Energy Agency; 1996.

26. International Atomic Energy Agency. The radiological accident in Istanbul. Vienna, Austria: International Atomic Energy Agency; 2000.

27. International Atomic Energy Agency. The radiological accident in Goiania. Vienna, Austria: International Atomic Energy Agency; 1998.

28. Ya-anant N, Tiyapun K, Saiyut K. Radiological accident and incident in Thailand: lesson to be learned. Radiat Prot Dosimetry
$2011 ; 146: 111-4$.

29. Bouville A, Beck HL, Simon SL. Doses from external irradiation to Marshall Islanders from Bikini and Enewetak nuclear weapons tests. Health Phys 2010;99:143-56.

30. Chang WP, Chan CC, Wang JD. 60Co contamination in recycled steel resulting in elevated civilian radiation doses: causes and challenges. Health Phys 1997;73:465-72.

31. Liu OJ, Lu X, Zhao H, et al. Cytogenetic analysis in 16-year follow-up study of a mother and fetus exposed in a radiation accident in Xinzhou, China. Mutat Res 2013;755:68-72.

32. Lloyd DC, Edwards AA, Moquet JE, Guerrero-Carbajal YC. The role of cytogenetics in early triage of radiation casualties. Appl Radiat Isot 2000;52:1107-12.

33. Li Y, Shirley BC, Wilkins RC, Norton F, Knoll JH, Rogan PK. Radiation dose estimation by completely automated interpretation of the dicentric chromosome assay. Radiat Prot Dosimetry 2019;186:42-7.

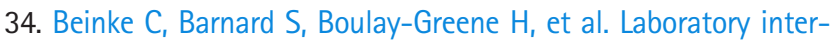
comparison of the dicentric chromosome analysis assay. Radiat Res 2013;180:129-37.

35. Wilkins RC, Romm H, Kao TC, et al. Interlaboratory comparison of the dicentric chromosome assay for radiation biodosimetry in mass casualty events. Radiat Res 2008;169:551-60.

36. Flegal FN, Devantier Y, Marro L, Wilkins RC. Validation of QuickScan dicentric chromosome analysis for high throughput radiation biological dosimetry. Health Phys 2012;102:143-53.

37. Fenech M, Morley A. Solutions to the kinetic problem in the micronucleus assay. Cytobios 1985;43:233-46.

38. Odagiri Y, Uchida H, Shibazaki S. Interindividual variation in cytogenetic response to $X$-ray and colchicine measured with the cytokinesis-block micronucleus assay. Mutat Res 1997;381:1-13.

39. Mikhalevich LS, De Zwart FA, Perepetskaya GA, Chebotareva NV, Mikhalevich EA, Tates AD. Radiation effects in lymphocytes of children living in a Chernobyl contaminated region of Belarus. Int J Radiat Biol 2000;76:1377-85.

40. International Atomic Energy Agency. Cytogenetic analysis for radiation dose assessment: a manual (Technical Report No. 405). Vienna, Austria: International Atomic Energy Agency; 2001, p. 71-87.

41. Vral A, Fenech $M$, Thierens $H$. The micronucleus assay as a biological dosimeter of in vivo ionising radiation exposure. Mutagenesis $2011 ; 26: 11-7$

42. McNamee JP, Flegal FN, Greene HB, Marro L, Wilkins RC. Validation of the cytokinesis-block micronucleus (CBMN) assay for use as a triage biological dosimetry tool. Radiat Prot Dosimetry 2009;135:232-42.

43. Depuydt J, Baeyens A, Barnard S, et al. RENEB intercomparison 
exercises analyzing micronuclei (Cytokinesis-block Micronucleus Assay). Int J Radiat Biol 2017;93:36-47.

44. Beinke $C_{1}$ Port $M$, Abend $M$. Automatic versus manual lymphocyte fixation: impact on dose estimation using the cytokinesis-block micronucleus assay. Radiat Environ Biophys 2015;54:81-90.

45. Rodrigues MA, Beaton-Green LA, Wilkins RC. Validation of the cytokinesis-block micronucleus assay using imaging flow cytometry for high throughput radiation biodosimetry. Health Phys 2016;110:29-36.

46. Rodrigues MA, Beaton-Green LA, Wilkins RC, Fenech MF. The potential for complete automated scoring of the cytokinesis block micronucleus cytome assay using imaging flow cytometry. Mutat Res Genet Toxicol Environ Mutagen 2018;836(Pt A):53-64.

47. Repin M, Pampou S, Garty G, Brenner DJ. RABiT-II: a fully-automated micronucleus assay system with shortened time to result. Radiat Res 2019;191:232-6.

48. Goh VS, Nakayama R, Blakely WF, et al. Improved harvest and fixation methodology for isolated human peripheral blood mononuclear cells in cytokinesis-block micronucleus assay. Int J Radiat Biol 2021;97:194-207.

49. Sreedevi B, Rao BS, Bhatt B. Radiation-induced chromosome aberration yields following an accidental non-uniform exposure. Radiat Prot Dosim 1993;50:45-9.

50. Ohtaki K, Shimba H, Awa AA, Sofuni T. Comparison of type and frequency of chromosome aberrations by conventional and G-staining methods in Hiroshima atomic bomb survivors. J Radiat Res 1982;23:441-9.

51. Kumagai E, Tanaka R, Kumagai T, Onomichi M, Sawada S. Effects of long-term radiation exposure on chromosomal aberrations in radiological technologists. J Radiat Res 1990;31:270-9.

52. Grégoire $E$, Roy $L$, Buard $V$, et al. Twenty years of FISH-based translocation analysis for retrospective ionizing radiation biodosimetry. Int J Radiat Biol 2018;94:248-58.

53. Lucas JN, Awa A, Straume T, et al. Rapid translocation frequency analysis in humans decades after exposure to ionizing radiation. Int J Radiat Biol 1992;62:53-63.

54. Guerrero-Carbajal YC, Moquet JE, Edwards AA, Lloyd DC. The persistence of FISH translocations for retrospective biological dosimetry after simulated whole or partial body irradiation. Radiat Prot Dosim 1998;76:159-68.

55. Darroudi F, Natarajan AT. Application of FISH chromosome painting assay for dose reconstruction: state of art and current views. Radiat Prot Dosim 2000;88:51-8.

56. Crespo RH, Domene MM, Rodriguez MJ. Biodosimetry and assessment of radiation dose. Rep Pract Oncol Radiother 2011;16:131-7.

57. Mazurik VK, Mikhailov VF, Ushenkova LN, et al. The comparison of molecular-biochemical and cytogenetic analyses of blood cells of patients long after the acute radiation sickness. Radiats Biol Radioecol 2006;46:393-409.

58. Sigurdson A, Ha M, Hauptmann M, et al. International study of factors affecting human chromosome translocations. Mutat Res 2008;652:112-21.

59. Johnson RT, Rao PN. Mammalian cell fusion: induction of premature chromosome condensation in interphase nuclei. Nature 1970;226:717-22.

60. Pantelias GE, Maillie HD. The use of peripheral blood mononuclear cell prematurely condensed chromosomes for biological dosimetry. Radiat Res 1984;99:140-50.

61. Rodriguez P, Barquinero JF, Duran A, Caballin MR, Ribas M, Barrios $\mathrm{L}$. Cells bearing chromosome aberrations lacking one telomere are selectively blocked at the $\mathrm{G} 2 / \mathrm{M}$ checkpoint. Mutat Res 2009:670:53-8.

62. Yao B, Li Y, Liu G, et al. Estimation of the biological dose received by five victims of a radiation accident using three different cytogenetic tools. Mutat Res 2013;751:66-72.

63. Pujol M, Barquinero JF, Puig P, Puig R, Caballin MR, Barrios L. A new model of biodosimetry to integrate low and high doses. PLoS One 2014;9:e114137.

64. Balakrishnan S, Shirsath K, Bhat N, Anjaria K. Biodosimetry for high dose accidental exposures by drug induced premature chromosome condensation (PCC) assay. Mutat Res 2010;699:11-6.

65. Lamadrid Al, Garcia O, Delbos M, Voisin P, Roy L. PCC-ring induction in human lymphocytes exposed to gamma and neutron irradiation. J Radiat Res 2007;48:1-6.

66. Romero I, Lamadrid Al, Gonzalez JE, Mandina T, Garcia O. Culture time and reagent minimization in the chemical PCC assay. Int J Radiat Biol 2016;92:558-62.

67. Lu X, Zhao H, Feng JB, Zhao XT, Chen DQ, Liu O. Dose response of multiple parameters for calyculin A-induced premature chromosome condensation in human peripheral blood lymphocytes exposed to high doses of cobalt-60 gamma-rays. Mutat Res Genet Toxicol Environ Mutagen 2016;807:47-54.

68. Hittelman WN, Rao PN. Premature chromosome condensation. I. Visualization of x-ray-induced chromosome damage in interphase cells. Mutat Res 1974;23:251-8.

69. Prasanna PG, Escalada ND, Blakely WF. Induction of premature chromosome condensation by a phosphatase inhibitor and a protein kinase in unstimulated human peripheral blood lymphocytes: a simple and rapid technique to study chromosome aberrations using specific whole-chromosome DNA hybridization probes for biological dosimetry. Mutat Res 2000;466:131-41.

70. Hayata I, Kanda R, Minamihisamatsu M, Furukawa M, Sasaki MS. Cytogenetical dose estimation for 3 severely exposed patients in 
the JCO criticality accident in Tokai-mura. J Radiat Res 2001;42 Suppl:S149-55.

71. M'kacher R, El Maalouf E, Terzoudi G, et al. Detection and automated scoring of dicentric chromosomes in nonstimulated lymphocyte prematurely condensed chromosomes after telomere and centromere staining. Int J Radiat Oncol Biol Phys 2015; 91:640-9.

72. Terzoudi GI, Pantelias G, Darroudi F, et al. Dose assessment intercomparisons within the RENEB network using G0-lymphocyte prematurely condensed chromosomes (PCC assay). Int J Radiat
Biol 2017;93:48-57.

73. Gonzalez JE, Romero I, Gregoire E, et al. Biodosimetry estimation using the ratio of the longest:shortest length in the premature chromosome condensation (PCC) method applying autocapture and automatic image analysis. J Radiat Res 2014;55:862-5.

74. Selvan Gnana Sekaran T, Ricoul M, Brochard P, Herate C, Sabatier $\mathrm{L}$. An alternative approach for the induction of premature chromosome condensation in human peripheral blood lymphocytes using mitotic Akodon cells. Int J Radiat Biol 2020;96:214-9. 